\title{
Metal traces in white dwarfs of the SPY (ESO Supernova la Progenitor Survey) sample ${ }^{\star}$
}

\author{
D. Koester ${ }^{1}$, K. Rollenhagen ${ }^{1}$, R. Napiwotzki ${ }^{2,3}$, B. Voss ${ }^{1}$, N. Christlieb ${ }^{4}$, D. Homeier ${ }^{5}$, and D. Reimers ${ }^{4}$ \\ ${ }^{1}$ Institut für Theoretische Physik und Astrophysik, University of Kiel, 24098 Kiel, Germany \\ e-mail: koester@astrophysik.uni-kiel.de \\ 2 Dept. of Physics \& Astronomy, University of Leicester, University Road, Leicester, LE1 7RH, UK \\ 3 Dr.-Remeis-Sternwarte, Astronomisches Institut der Universität Erlangen-Nürnberg, Sternwartstr. 7, 96049 Bamberg, \\ Germany \\ ${ }^{4}$ Hamburger Sternwarte, Gojenbergsweg 112, 21029 Hamburg, Germany \\ 5 Department of Physics and Astronomy, The University of Georgia, Athens, GA 30602-2451, USA
}

Received 30 August 2004 / Accepted 5 November 2004

\begin{abstract}
We search for faint Ca II lines in the spectra of about 800 apparently single white dwarfs observed at high resolution for the SPY (ESO Supernova Progenitor Survey) survey. Photospheric Ca is detected in 24 DAZ; in 25 mostly hot objects the observed lines must be interstellar. We also rediscover 9 strong $\mathrm{H}$ and $\mathrm{K}$ lines in helium-dominated atmospheres and discover for the first time faint lines in 8 DB. Most of these also show faint hydrogen lines and are thus classified DBAZ. The distribution of metal abundances is discussed and compared with the predictions of the accretion/diffusion scenario. We argue that the observations are easier to understand in a scenario of continuous ongoing accretion with rates varying with the conditions of the ambient medium, rather than with the strongly idealized "two phase accretion/diffusion scenario" of Dupuis et al. (1992, 1993a,b).
\end{abstract}

Key words. stars: white dwarfs - stars: atmospheres - stars: abundances

\section{Introduction}

Traces of heavy elements have been known in white dwarfs with helium-dominated atmospheres for many decades. Most prominent is the $\mathrm{Ca}$ II $\mathrm{K}$ resonance line of calcium, but $\mathrm{Mg}$ and Fe are also sometimes found, especially if UV spectroscopy is available. If the effective temperatures are high enough for the HeI lines to become visible the spectral type is DBZ, if the temperatures are too low and the metal lines are the only visible features the type is called DZ. In the latter case the major atmospheric constituent $(\mathrm{H}$ or $\mathrm{He})$ is not obvious and can only be determined by a careful analysis. At higher temperatures (approximately $7000 \mathrm{~K}$ ) the distinction is possible and the vast majority of DA - defined by the presence of the Balmer series - is indeed H-rich. This much larger class of hydrogen-dominated white dwarfs had for many years only one certain object with metal lines, G74-4, the prototype of the DAZ class (white dwarfs with dominating hydrogen lines and additional metal lines). Two more members were detected by Koester et al. (1997) and Holberg et al. (1997), but a real breakthrough came with the work of Zuckerman \& Reid (1998) and

* Based on data obtained at the Paranal Observatory of the European Southern Observatory for programs 165.H-0588 and 167.D-0407.
Zuckerman et al. (2003) (henceforth Z98 and Z03), which increased the number of known DAZ to approximately 20. It has become clear now that the difference is simply due to observational bias: because of the much higher opacity of hydrogen in the temperature range 6000 to $20000 \mathrm{~K}$ the equivalent widths of Ca II lines are a factor of 100 or more smaller at the same metal abundance in hydrogen atmospheres than in helium-dominated atmospheres. High-resolution spectroscopy at very large telescopes is therefore needed to detect the lines.

Nevertheless the effort is worthwhile. The most widely accepted explanation for the presence of metals is accretion from interstellar matter. The lifetime of the heavy elements against diffusion out of the atmosphere or outer convective layers into deeper regions is very short compared to the cooling age of the white dwarfs, and thus the elements cannot be primordial. The accretion-diffusion scenario, which was developed from earlier suggestions by many authors in a series of three fundamental papers by Dupuis et al. (1992, 1993a,b), is not without serious problems, as discussed in detail in Z03. Perhaps the most difficult problem is the lack of any correlation between objects with metals and the conditions of the interstellar matter from which the metals are presumably accreted (Aannestad et al. 1993). In this respect the new study of the DAZ is promising since the diffusion time scales in a DAZ with $T_{\text {eff }}$ around $10000 \mathrm{~K}$ are 


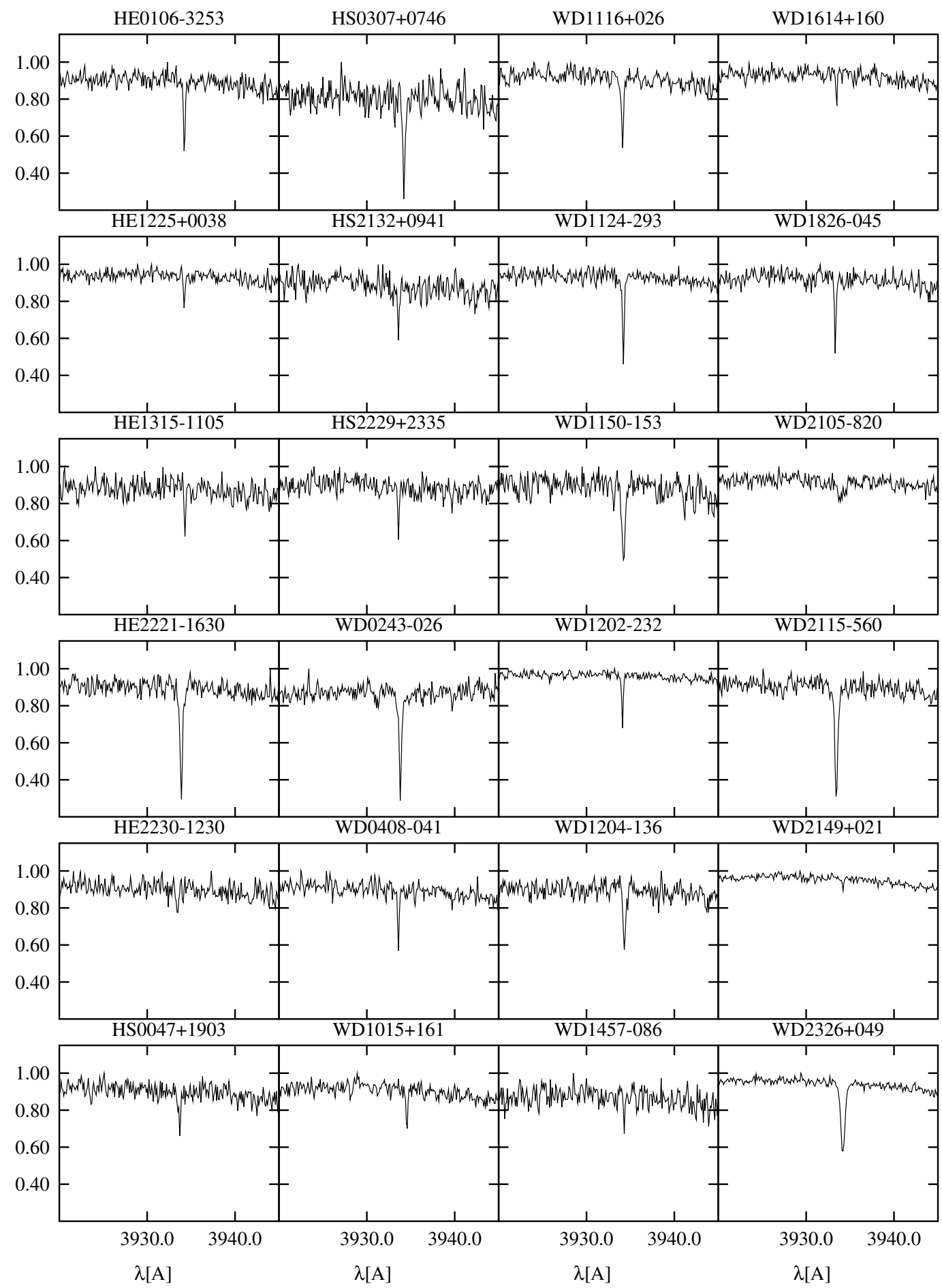

Fig. 1. Spectra of 24 DAZ identified in the sample, of which 6 had been found previously (see Table 1). In most cases, and in particular for WD2105-820, HE2230-1230, WD2149+021 with very small and uncertain features, at least one additional spectrum exists with similar absorption features at the correct wavelength, making the identifications plausible.

only $10^{3} \mathrm{yr}$ as compared to $\approx 10^{6} \mathrm{yr}$ in a DZ (Dupuis et al. 1992). The DAZ should therefore be accreting right now as we observe it or in any case still be very close to the place where accretion occurred. A DZ on the contrary could have traveled many parsec away from the accretion episode. The DAZ therefore promise to become a more efficient tool for the study of the relation between interstellar matter and accretion rates. In this paper we report on an extension of the search using a sample from the southern hemisphere observed with the VLT, which doubles the number of known DAZ.

\section{Observations}

The spectra of our sample were obtained as part of the search for close double degenerate binary systems in the ESO SN Ia progenitor survey (=SPY, Napiwotzki et al. 2001, 2003). 
A first part of about 200 white dwarfs of the total sample was analyzed by Koester et al. (2001). The selection of the sample, the specific nature of the search as a "filler project" for mediocre weather conditions, and the reduction procedures are described in the first paper. We therefore repeat here only the most important characteristics.

The spectra were obtained with the UV-Visual Echelle Spectrograph (UVES) at the Unit 2 Telescope (Kueyen) of the Very Large Telescope of ESO on Paranal. The slit width is 2.1" leading to a resolution of about 18500 or better, depending on the seeing. The $\mathrm{S} / \mathrm{N}$ per binned pixel of $0.1 \AA$ used in the present work is usually 15 or higher.

In the meantime the survey has been almost finished and the present sample consists of approximately 1700 spectra for about 1000 objects. The main emphasis in this paper is on the search for weak Ca II lines in DA white dwarfs of the sample, but we also report briefly on some results for helium-rich white dwarfs. Atmospheric parameters $\left(T_{\mathrm{eff}}, \log g\right)$ were determined for most objects using the "analysis pipeline" described in Koester et al. (2001) with spectra binned to $0.4 \AA$. A detailed analysis using the spectra binned to $0.1 \AA$, which were used for the search for metals, is in preparation (Voss et al. 2005), but was not yet available for this work.

\section{Automated search for the Ca II $\mathrm{K}$ line and measurements}

Because of the very large amount of data we have developed automatic search routines to select a smaller sample for more detailed study. Identification of a Ca II K line employs three methods

- Direct integration of equivalent width, assuming that a line is present near the laboratory wavelength of $3933.661 \AA$. The continuum is placed to the left and right of this position using the median of the flux in predefined intervals. The error of the equivalent width is calculated by propagating all errors from $\mathrm{S} / \mathrm{N}$ of the spectrum and the placement of the continuum.

- Fitting a Gaussian profile function to an interval around the Ca II K wavelength with central wavelength, line width, and central depth as free parameters. The equivalent width is calculated from the fit parameters, its error from the errors of the fit parameters as determined by the $\chi^{2}$-fitting routine.

- Similar to the second method but using a Lorentzian profile function.

The probability of a real line being present was determined by a combination of all three results with some weighting determined from empirical tests. Typically the criteria included that the equivalent width should be larger than the error and the line depth at least 5 times the $1 \sigma$ error of nearby continuum points. All spectra where a line was suspected were then individually inspected by eye. If the line was confirmed the equivalent width and Doppler velocity from the position of the line center were measured. For the confirmed lines the equivalent widths and line positions determined by the different methods usually agreed within the errors. As could be expected, however, the smallest lines are best fit with a Gaussian, and the strongest lines with a Lorentzian profile. We also determined the Doppler velocities from the Balmer lines contained in the spectral regions by fitting Lorentz profiles to the inner cores of the broad lines. For the final comparison between radial velocities from $\mathrm{Ca}$ lines and from hydrogen lines we used the hydrogen velocities determined by R.N. in the SPY search for variable velocities.

Fifteen additional (mostly hot) objects with very weak $\mathrm{Ca}$ lines were discovered by visual inspection in the course of the complete reanalysis mentioned above, and added to our sample.

From the equivalent widths the $\mathrm{Ca}$ abundances were determined using the $T_{\text {eff }}$ and $\log g$ from the preliminary fits described above and the same table of equivalent widths vs. abundances used in Z03. If no line is detected an upper limit is determined using the (spurious) equivalent width plus its $1 \sigma$ error.

\section{The DAZ and their $\mathrm{Ca}$ abundances}

$\mathrm{Ca}$ II lines in the spectra may indicate photospheric Ca, but may also be due to interstellar absorption. This is more likely for the hotter objects. The resonance lines should become unobservable above approximately $25000 \mathrm{~K}$ because of the ionization to Ca III; in addition the hotter stars tend to be farther away from the sun. We have used two criteria to select those objects where the metals are very likely photospheric: $T_{\text {eff }}$ below $25000 \mathrm{~K}$ and the difference between the radial velocities from $\mathrm{Ca}$ and the Balmer lines should be comparable to their combined $1 \sigma$ errors. This "photospheric" sample includes 24 objects and is given in Table 1. We recover 6 objects in this table, which have been discovered as DAZ before by Z98 or Z03; they are marked as "DAZ"; the other 18 objects are new detections.

Table 2 contains 25 objects, where the Ca lines must clearly be interstellar because of the high $T_{\text {eff }}$ or a large discrepancy in radial velocities.

We also find in our sample 454 apparently normal DA below $T_{\text {eff }} \approx 30000 \mathrm{~K}$ with no indication for metals, for which we have atmospheric parameter determinations. For these we determine upper limits for $\mathrm{Ca} / \mathrm{H}$ as described above. Figure 2 shows the results for these 478 objects. The most notable effect in this figure is the apparent increase of abundances and upper limits with temperature, which is of course a result of the decreasing line strength of the $\mathrm{Ca}$ line because of increasing ionization of Ca II. This is demonstrated by the solid line, which gives the locus of constant equivalent width of $15 \mathrm{~m} \AA$ according to our theoretical models (with $\log g=8.0$ ). This is the approximate visibility limit for our best spectra; with decreasing $\mathrm{S} / \mathrm{N}$ the limit moves upwards to higher $E W$.

We also note that the fraction of DA showing $\mathrm{Ca}$ is much smaller than the value of $20-25 \%$ found in Z03. This is a selection effect due to the different nature of the two samples. While the Z03 sample was chosen to be bright stars (for high $\mathrm{S} / \mathrm{N}$ ) and mostly below $10000 \mathrm{~K}$ for the greatest chance to detect metals, the SPY sample was selected without this project in mind and is dominated by hotter white dwarfs mostly above $10000 \mathrm{~K}$ (Napiwotzki et al. 2001). As Fig. 2 demonstrates, only the highest $\mathrm{Ca}$ abundances can be detected at the higher $T_{\text {eff }}$. In addition 
Table 1. Hydrogen-rich white dwarfs with atmospheric Ca. Given are the equivalent width of the Ca II K line, atmsopheric parameters $T_{\text {eff }}$ and $\log g$, and the derived $\mathrm{Ca}$ abundance as the logarithmic number ratio. The radial velocities are corrected to heliocentric values; the errors are $1 \sigma$ errors; the Balmer velocities $v_{\mathrm{H}}$ are averages from $\mathrm{H} \alpha$ and $\mathrm{H} \beta$. In the objects marked DAZ the Ca was originally detected by Z98 or Z03.

\begin{tabular}{lrrrrrrr}
\hline \hline Object & $E W[\mathrm{~m} \AA]$ & $T_{\text {eff }}[\mathrm{K}]$ & $\log g$ & {$[\mathrm{Ca} / \mathrm{H}]$} & $v_{\mathrm{H}}\left[\mathrm{km} \mathrm{s}^{-1}\right]$ & $v_{\mathrm{Ca}}\left[\mathrm{km} \mathrm{s}^{-1}\right]$ & Remarks \\
\hline HS 0047+1903 & 270 & 16600 & 7.8 & -5.6 & $25 \pm 2$ & $24 \pm 3$ & \\
HE 0106-3253 & 107 & 15700 & 8.0 & -6.4 & $57 \pm 1$ & $55 \pm 1$ & \\
WD 0243-026 & 219 & 6800 & 8.2 & -9.9 & $30 \pm 2$ & $30 \pm 2$ & DAZ \\
HS 0307+0746 & 223 & 10200 & 8.1 & -7.6 & $13 \pm 2$ & $13 \pm 2$ & \\
WD 0408-041 & 77 & 14400 & 7.8 & -7.1 & $21 \pm 2$ & $19 \pm 1$ & \\
WD 1015+161 & 58 & 19300 & 7.9 & -6.3 & $65 \pm 2$ & $67 \pm 2$ & \\
WD 1116+026 & 135 & 12200 & 7.9 & -7.3 & $47 \pm 1$ & $46 \pm 1$ & \\
WD 1124-293 & 118 & 9700 & 8.1 & -8.5 & $30 \pm 1$ & $29 \pm 1$ & DAZ \\
WD 1150-153 & 208 & 12800 & 7.8 & -6.7 & $25 \pm 2$ & $22 \pm 2$ & \\
WD 1202-232 & 58 & 8800 & 8.2 & -9.7 & $21 \pm 1$ & $23 \pm 1$ & DAZ \\
WD 1204-136 & 125 & 11200 & 8.0 & -7.7 & $37 \pm 3$ & $36 \pm 2$ & DAZ \\
HE 1225+0038 & 38 & 9400 & 8.1 & -9.7 & $12 \pm 1$ & $13 \pm 2$ & \\
HE 1315-1105 & 74 & 9400 & 8.4 & -9.2 & $33 \pm 1$ & $33 \pm 1$ & \\
WD 1457-086 & 45 & 20400 & 8.0 & -6.3 & $22 \pm 2$ & $19 \pm 2$ & \\
WD 1614+160 & 31 & 17400 & 7.8 & -7.2 & $-24 \pm 1$ & $-26 \pm 3$ & \\
WD 1826-045 & 88 & 9200 & 8.1 & -9.1 & $1 \pm 2$ & $-1 \pm 1$ & DAZ \\
WD 2105-820 & 80 & 10300 & 8.0 & -8.6 & $42 \pm 3$ & $45 \pm 3$ & \\
WD 2115-560 & 294 & 9700 & 8.1 & -7.6 & $4 \pm 2$ & $5 \pm 1$ & \\
HS 2132+0941 & 66 & 13200 & 7.7 & -7.7 & $-4 \pm 2$ & $-6 \pm 3$ & \\
WD 2149+021 & 15 & 17300 & 7.9 & -7.6 & $28 \pm 1$ & $26 \pm 3$ & \\
HE 2221-1630 & 231 & 10100 & 8.2 & -7.6 & $45 \pm 3$ & $45 \pm 1$ & \\
HS 2229+2335 & 62 & 18600 & 7.9 & -6.3 & $-12 \pm 2$ & $-12 \pm 1$ & \\
HE 2230-1230 & 47 & 20300 & 7.7 & -6.3 & $14 \pm 2$ & $11 \pm 3$ & \\
WD 2326+049 & 238 & 12100 & 7.9 & -6.8 & $42 \pm 2$ & $41 \pm 1$ & DAZ \\
\hline
\end{tabular}

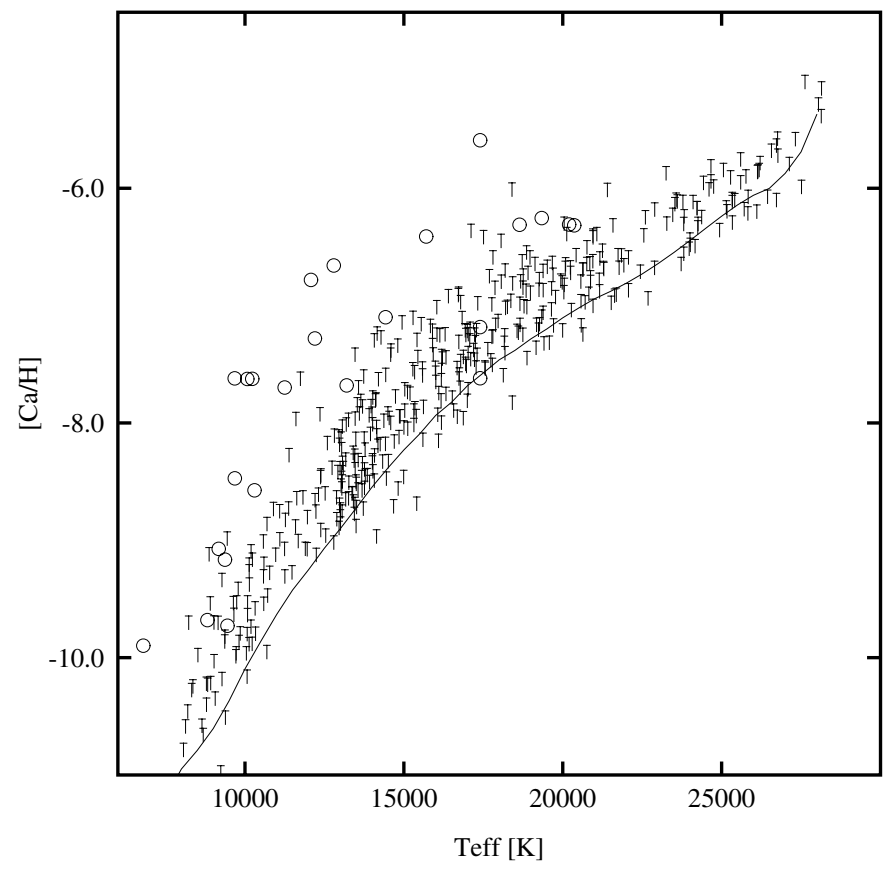

Fig. 2. Logarithmic $\mathrm{Ca}$ abundances $[\mathrm{Ca} / \mathrm{H}]$ for 24 and upper limits for 454 DA white dwarfs of the SPY sample. Open circles are positive detections, the other symbols are upper limits. The solid line indicates a line of constant equivalent width of $15 \mathrm{~m} \AA$, which is approximately the detection limit for the spectra with highest $\mathrm{S} / \mathrm{N}$. the resolution in Z03 was 34000 in the blue compared to 18500 in the SPY sample, and the S/N on average was slightly higher.

\section{Results on helium-rich objects}

The SPY sample contains a significant fraction of white dwarfs without (strong) hydrogen Balmer lines. Among these we recover 9 objects with strong $\mathrm{Ca}$ II $\mathrm{H}$ and $\mathrm{K}$ lines which have been known and described before in the literature (see McCook \& Sion 1999, for more information on individual objects). If $\mathrm{He}$ lines are visible the stars must be hotter than about $10000 \mathrm{~K}$ and the atmospheres are helium-dominated; the spectral type is DBZ. If no $\mathrm{H}$ or He lines are visible the spectral type is $\mathrm{DZ}$ and as mentioned in the introduction the atmospheric composition is uncertain.

In addition we find weaker $\mathrm{Ca}$ lines, comparable to those in the DAZ, in $8 \mathrm{DB}$ white dwarfs. All but two of these also show hydrogen lines, changing the classification to DBAZ (and DBZ for the other two); HE 0110-5630 is the only new white dwarf among these that has no previously published classification. Within the (rather large) errors of the radial velocities determined from $\mathrm{He}$ I lines they agree with the Ca II line velocity. We thus assume that the lines are photospheric.

If the origin of the hydrogen and calcium is accretion from interstellar matter (see discussion below), hydrogen as the lightest element should remain within the atmosphere or 
Table 2. Hydrogen-rich white dwarfs with interstellar Ca lines. Radial velocities for hydrogen (average of $\mathrm{H} \alpha$ and $\mathrm{H} \beta$ ) and $\mathrm{Ca}$ are corrected to heliocentric

\begin{tabular}{|c|c|c|c|c|c|c|}
\hline Object & $E W[\mathrm{~m} \AA]$ & $T_{\text {eff }}[\mathrm{K}]$ & $\log g$ & $v_{\mathrm{H}}\left[\mathrm{km} \mathrm{s}^{-1}\right]$ & $v_{\mathrm{Ca}}\left[\mathrm{km} \mathrm{s}^{-1}\right]$ & Remarks \\
\hline WD 0027-636 & 48 & 59100 & 7.9 & 31 & 5 & \\
\hline WD 0205-136j & 50 & 44600 & 7.8 & 15 & 0 & \\
\hline WD 0209+085 & 31 & 37300 & 8.0 & 72 & 82 & \\
\hline HE 0210-2012 & 40 & 16700 & 7.8 & 37 & 17 & 1 \\
\hline WD $0328+008$ & 93 & 34300 & 7.9 & 21 & 14 & \\
\hline WD 0330-009 & 49 & 34100 & 7.8 & -2 & 18 & \\
\hline HS 0424+0141 & 62 & 43500 & 7.5 & 57 & 23 & 2 \\
\hline WD 0443-037j & 49 & 69700 & 8.3 & 145 & -27 & \\
\hline HS $0503+0154$ & 35 & 60600 & 7.6 & 5 & 26 & \\
\hline WD $0548+000$ & 34 & 47100 & 7.9 & 89 & 27 & \\
\hline WD 1144-246 & 54 & 30200 & 7.0 & -8 & 4 & \\
\hline EC 13123-2523 & 96 & 60300 & 7.0 & 25 & -6 & \\
\hline WD 1328-152 & 31 & 63400 & 7.7 & 9 & -9 & 2 \\
\hline WD $1755+194$ & 62 & 24700 & 7.8 & 45 & -26 & 3 \\
\hline HS 2046+0044 & 54 & 26600 & 7.8 & 23 & -11 & \\
\hline WD $2120+054$ & 54 & 35200 & 7.8 & 9 & -9 & \\
\hline WD $2127-221 j$ & 44 & 45500 & 7.7 & -2 & -10 & \\
\hline WD 2146-433 & 49 & 71500 & 7.1 & 42 & 31 & \\
\hline WD $2150+021$ & 51 & 42300 & 7.6 & $21 /-92$ & -11 & 4 \\
\hline WD 2240-045 & 45 & 41800 & 7.8 & -71 & -8 & \\
\hline HS 2244+0305 & 73 & 61300 & 7.6 & -48 & -10 & \\
\hline WD $2303+017$ & 82 & 43000 & 7.8 & 46 & -7 & \\
\hline WD $2308+050$ & 48 & 36300 & 7.6 & 26 & -7 & \\
\hline WD $2353+026$ & 38 & 65700 & 7.7 & 0 & -1 & \\
\hline WD 2354-151 & 41 & 34600 & 7.2 & 19 & -8 & \\
\hline
\end{tabular}

Remarks:

1: Ca line clearly visible only in one of two spectra. Although HE 0210-2012 is significantly cooler than our limit of $25000 \mathrm{~K}$ the difference between Balmer and $\mathrm{Ca}$ line velocity is larger than the errors and the $\mathrm{Ca}$ is probably interstellar.

2: Ca line clearly visible only in one of two spectra.

3: WD $1755+194$ is slightly cooler than our limit of $25000 \mathrm{~K}$; the discrepancy between Balmer and Ca line velocities is much larger than the errors and the Ca must be interstellar.

4: Double degenerate.

outer convection zone, whereas $\mathrm{Ca}$ is expected to diffuse downward. The abundance ratio $\log \mathrm{Ca} / \mathrm{H}$ should therefore be always smaller than or at most equal to that in the accreted matter. The observed ratio (see Table 3 ) ranges from -4.6 to -3.1 , always larger than the solar ratio (-5.7). This indicates that the abundances in the accreted matter are not solar, a well-known result for many DB and DBZ stars with traces of hydrogen.

\section{Results and discussion}

Taking together the present and the Z03 positive detections in apparently single DA we have parameters and $\mathrm{Ca}$ abundances for $41 \mathrm{DAZ}$. These are shown - together with the data for $7 \mathrm{bi}$ naries from Z03 - in Fig. 4. With the exception of one object, there seems to be an upper limit to the abundance around $[\mathrm{Ca} / \mathrm{H}]=-6.5$, which is approximated by several objects with $T_{\text {eff }}=12000-20000 \mathrm{~K}$. Below $T_{\text {eff }}=10000 \mathrm{~K}$ the maximum observed abundance decreases, very likely reflecting the increasing depth of the convection zone and the dilution of the accreted matter within a much larger mass. The lower limit is set by the observational limits, strongly temperature-dependent due to the ionization of $\mathrm{Ca}$ II to Ca III.

In the introduction we discussed the expectation that the DAZ may allow a much more stringent test of the accretion/diffusion scenario because the short lifetimes of the heavy element abundances at the surface should facilitate any study of correlations between metal abundances and the local conditions of the interstellar matter. We will try here to explore some conclusions from our results with some very simple arguments. As a basis we use the scenario and results of the three Dupuis et al. papers (Dupuis et al. 1992, 1993a,b). It should be clear, however, and has been stated very clearly in those papers, that this scenario is highly idealized and needs refinement when our knowledge about the nature of the local ISM advances. There are no calculations for DA hotter than $10000 \mathrm{~K}$ in those papers. We are currently extending similar calculations of diffusion 
Table 3. Helium-rich white dwarfs with $\mathrm{Ca}$ II lines. Above the line are previously known objects with strong $\mathrm{H}$ and $\mathrm{K}$ lines, and the equivalent widths $(E W)$ are for both lines of the doublet combined, except for those marked with K. DBAZ is the spectral type for white dwarfs with helium-rich atmospheres and weaker hydrogen and metal lines. The $\mathrm{Ca}$ abundances are taken from the literature, with the codes (in column remarks) D93: Dupuis et al. (1993b) and original references therein, W02: Wolff et al. (2002), F00: Friedrich et al. (2000), F99: Friedrich et al. (1999), Z03: Zuckerman et al. (2003). In the lower half of the table are weaker metal lines discovered in this work. H $\alpha, \mathrm{H} \beta$ in the remark column indicate the visible lines, with the $E W$ in $\AA$ in parentheses.

\begin{tabular}{|c|c|c|c|c|c|c|c|}
\hline Object & $E W[\mathrm{~m} \AA]$ & $T_{\mathrm{eff}}[\mathrm{K}]$ & $\log g$ & {$[\mathrm{Ca} / \mathrm{He}]$} & {$[\mathrm{H} / \mathrm{He}]$} & Sp. type & Remarks \\
\hline HE 0449-2554 & 20000 & 12200 & 8.20 & -7.2 & & DBAZ & $\mathrm{F} 00, \mathrm{H} \alpha(2.8)$ \\
\hline WD $0046+051$ & 34400 & 5700 & 7.90 & -10.7 & & DZ & W02 \\
\hline WD 0300-013 & K2700 & 15200 & 8.00 & -6.7 & & DBZA & F99 \\
\hline WD 0322-019 & $\mathrm{K} 2500$ & 5200 & 8.00 & -11.4 & & DZA & $\mathrm{Z} 03, \mathrm{H} \alpha(2.7)$ \\
\hline WD 1225-079 & 39000 & 10500 & 8.00 & -7.9 & & DZAB & W02 H $\alpha(10.3), \mathrm{H} \beta, \mathrm{H} \gamma, \mathrm{He} 5875$ \\
\hline WD $1705+030$ & 42000 & 6200 & 8.00 & -10.2 & & DZ & D93 \\
\hline WD 2216-657 & 37000 & 9200 & 8.00 & -9.1 & & $\mathrm{DZ}$ & W02 \\
\hline WD 2312-024 & 53300 & 6000 & 8.00 & -10.5 & & DZ & D93 \\
\hline WD $2322+118$ & 25000 & 12000 & 8.00 & -8.7 & & DZA & $\mathrm{D} 93, \mathrm{H} \alpha(6.5), \mathrm{H} \beta$ \\
\hline HE $0110-5630$ & 130 & 19200 & 8.16 & -7.9 & -4.2 & DBAZ & $\mathrm{H} \alpha(2.3), \mathrm{H} \beta$ \\
\hline HE 1349-2305 & 180 & 18300 & 8.13 & -8.0 & -4.9 & DBAZ & $\mathrm{H} \alpha(1.0)$ \\
\hline WD 1352+004 & 150 & 15300 & 8.48 & -9.3 & -4.8 & DBAZ & $\mathrm{H} \alpha(2.0), \mathrm{H} \beta$ \\
\hline WD $1709+230$ & 75 & 19700 & 8.17 & -8.0 & -4.2 & DBAZ & $\mathrm{H} \alpha(2.0), \mathrm{H} \beta$ \\
\hline WD 2142-169 & 100 & 15900 & 7.93 & -9.1 & -4.5 & DBAZ & $\mathrm{H} \alpha(2.6), \mathrm{H} \beta$ \\
\hline WD 2144-079 & 140 & 16500 & 7.90 & -8.5 & & DBZ & \\
\hline WD 2229+139 & 140 & 15900 & 8.37 & -9.0 & -4.5 & DBAZ & $\mathrm{H} \alpha(2.6), \mathrm{H} \beta$ \\
\hline WD 2354+159 & 80 & 19000 & 7.91 & -8.1 & & DBZ & \\
\hline
\end{tabular}

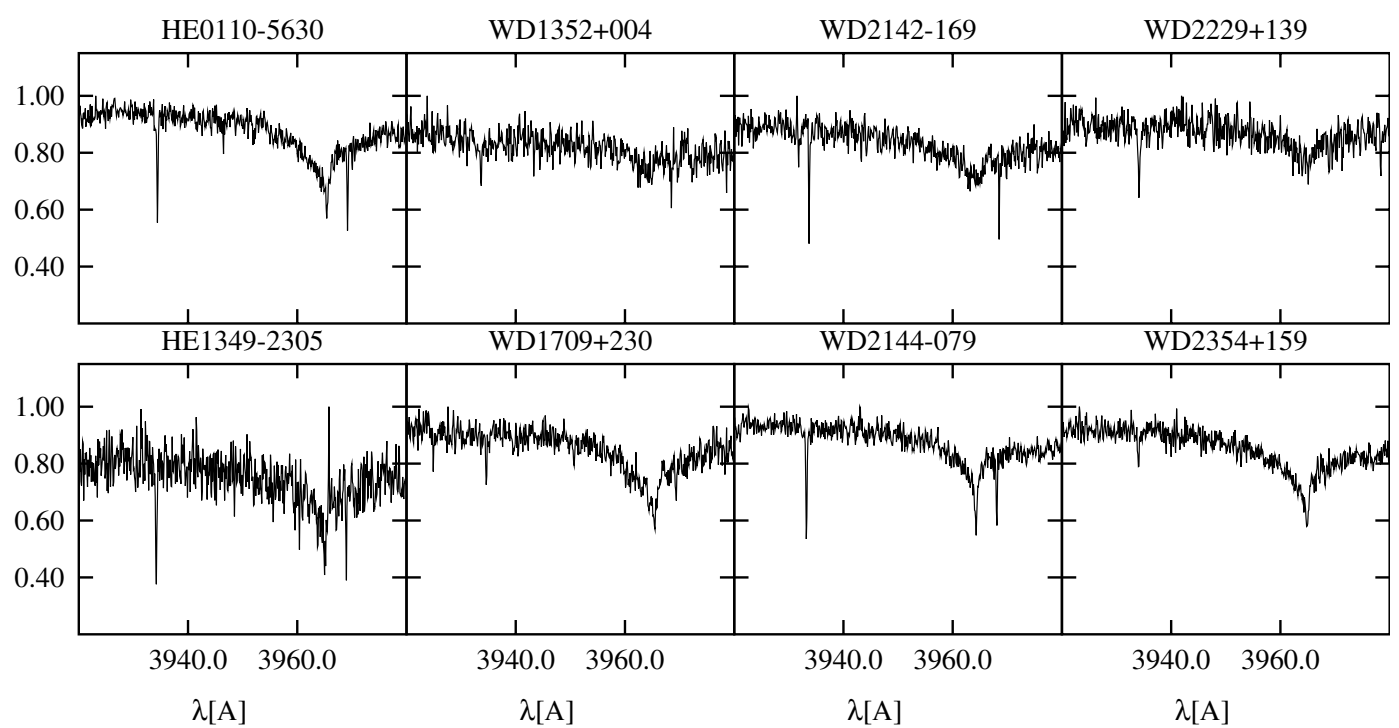

Fig. 3. Spectra of newly identified DBZ in the SPY sample. (see Table 3).

time scales and equilibrium abundances to hotter DA, but for this discussion we will concentrate on the $10000 \mathrm{~K}$ range and use estimates derived from the Dupuis et al. work. Using their Fig. 13 in the first paper, we find that the Ca surface abundance in a DA at $10000 \mathrm{~K}$ drops by 25 orders of magnitude in $7 \times$ $10^{4} \mathrm{yr}$. This translates into a diffusion time scale $\tau_{\mathrm{d}}=10^{3} \mathrm{yr}$, assuming a simple exponential decay.

From our observations in Fig. 2 we estimate an upper limit of -7.5 and a lower (observational) limit of -10.5 for the logarithmic abundances at $10000 \mathrm{~K}$. If the upper limit corresponds to the steady state abundance for a white dwarf crossing a dense cloud of interstellar matter, we would expect the abundance to fall below the observational limit approximately $7 \times$ $10^{3} \mathrm{yr}$ after exit from the cloud, and we would expect the region between upper and lower limit to be homogeneously populated (exponential decay leads to constant times for one decade of the abundance). While the latter conclusion is supported by the observations, taking the Dupuis scenario literally we would expect many more objects at the upper limit, since the cloud crossing time - during which the abundances should stay constant at the steady state value - is assumed to be $10^{6} \mathrm{yr}$. Another conclusion would be that the percentage of DA with 


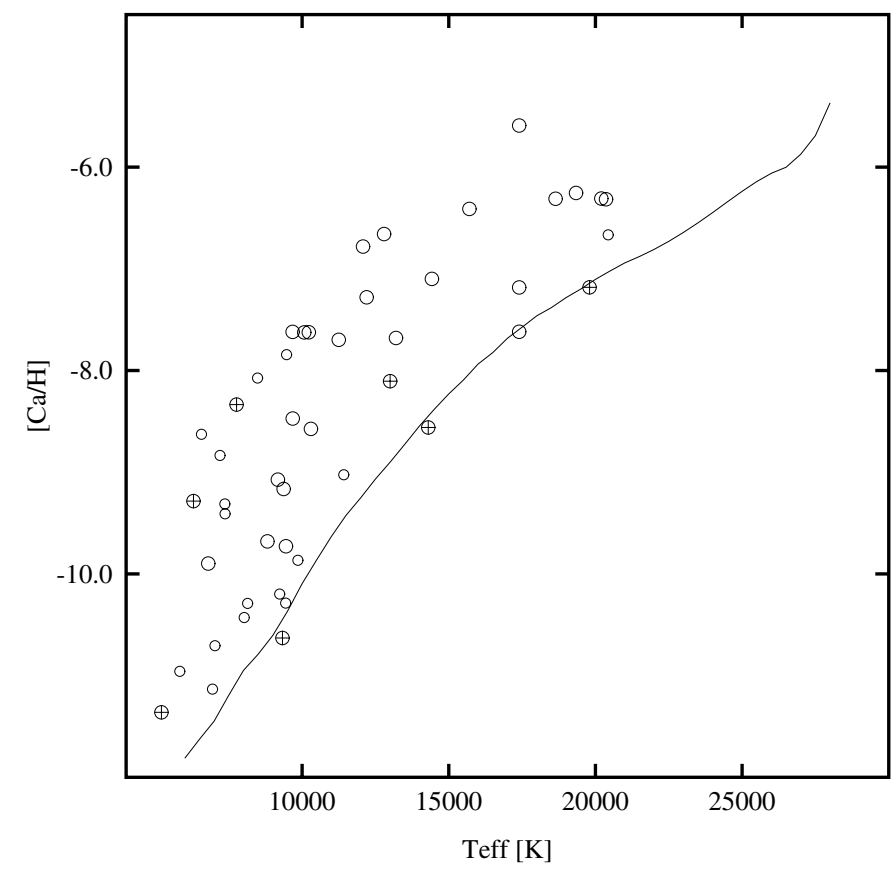

Fig. 4. Logarithmic $\mathrm{Ca}$ abundances $[\mathrm{Ca} / \mathrm{H}]$ for the combined sample from this work (larger circles) with the results from Z03 (smaller circles). Included from the latter paper are the white dwarfs known or suspected to be in binary systems (circles with + sign). Also shown is the same line of constant equivalent width from Fig. 2.

observable metals should approximate the ratio of the cloud crossing time to the time spent between encounters, that is 1:50. This is clearly at odds with the observations, which find a much larger fraction of DAZ (more than $20 \%$ in Z03).

Another estimate which can be derived from the short visibility of the metals is that a typical white dwarf with a space velocity of $20 \mathrm{~km} \mathrm{~s}^{-1}$ can only have traveled about $0.13 \mathrm{pc}$ since the end of the accretion episode. This confirms again the statement made in the introduction, that the DAZ must still be very close to the place where accretion occurred.

If we define the accretion time scale for a specific element as the time it takes to replace all ions of that element in the outer convection zone, we find

$\tau_{\mathrm{acc}}=\frac{\Delta M_{\mathrm{cz}} X}{\dot{M} X_{\odot}}$

with the mass in the convection zone $\Delta M_{\mathrm{cz}}$ in solar masses, accretion rate $\dot{M}$ in solar masses per year, $X$ the starting abundance of the element in the convection zone, and solar abundance $X_{\odot}$ for the element in the accreted matter. If a steady state between accretion and diffusion is reached, the accretion time scale is equal to the diffusion time scale $\tau_{\mathrm{d}}$ and the steady state abundance $X_{\mathrm{ss}}$ in the outer layers (either convection zone or atmosphere in the absence of convection) is given by (see Eq. (4) in Dupuis et al. 1993a)

$X_{\mathrm{ss}}=\frac{\tau_{\mathrm{d}} \dot{M} X_{\odot}}{\Delta M_{\mathrm{cz}}}$.

Using estimates for a $0.6 M_{\odot}$ DA white dwarf we find the necessary accretion rates for the upper abundance limit of
$[\mathrm{Ca} / \mathrm{H}]=-7.5$ to be $\dot{M}=10^{-15} M_{\odot} \mathrm{yr}^{-1}$. For the lower limit $([\mathrm{Ca} / \mathrm{H}]=-10.5)$ we find $\dot{M}=10^{-18} M_{\odot} \mathrm{yr}^{-1}$. These numbers are completely consistent with the numbers derived by Dupuis et al. for the accreting helium-rich white dwarfs.

Can these accretion rates be sustained by the conditions of the local ISM? Our knowledge is far from complete, though promising advances are currently being made through the work of Redfield \& Linsky (2002, 2004); Lehner et al. (2003) and others. The emerging picture is that the Local Bubble (within $70 \mathrm{pc}$ ) contains very little cold gas in the form of $\mathrm{HI}$ or $\mathrm{H}_{2}$. The dominant phase of the ISM is the warm phase with typical temperatures of about $7000 \mathrm{~K}$. Though the distribution of this gas is not yet known in much detail, Redfield \& Linsky (2004) are able to distinguish from 1 to 3 individual components in the absorption lines of many different ions along the lines of sight. Assuming a typical H I density of $0.1 \mathrm{~cm}^{-3}$ they derive typical length scales for these clouds between 0.1 and $11 \mathrm{pc}$, with a mean value of $2.2 \mathrm{pc}$. With a very simple calculation, assuming that all the clouds within $50 \mathrm{pc}$ (where most of their targets are located) cover the solid angle of the whole sky twice, we find a filling factor ranging from $0.5 \%$ to $50 \%$, a wide range covering the observed fractions of DAZ vs. DA. However, the crossing times for such clouds would still be large compared to the visibility times after the cloud encounter, in conflict with the homogenous distribution of abundances.

This problem leads us to consider an alternative scenario, in which the abundances in all observed objects are considered as steady state abundances for ongoing accretion in slightly different ambient conditions. If we take the "cloud sizes" discussed by Redfield \& Linsky (2004) as the typical scale for changes in LISM conditions the time scale connected with that would be larger than $10^{5} \mathrm{yr}$ for a white dwarf traveling at $20 \mathrm{~km} \mathrm{~s}^{-1}$, much larger than the diffusion time scale and enough to reach approximately steady state. As shown above, the accretion rates necessary to explain the observed abundances would be $10^{-15}-10^{-18} M_{\odot} \mathrm{yr}^{-1}$. Koester (1976) and Wesemael (1979) have argued that the Hoyle-Bondi accretion formula is not applicable at the low densities of the warm ISM phase and that therefore accretion rates would be insignificant. This has, however, been disputed by Alcock \& Illarionov (1980), who argue that in a partially ionized plasma interactions are always strong enough to lead to accretion close to the Hoyle-Bondi value. If we follow their arguments the accretion rate is

$\dot{M}=\frac{2.5 \pi(G M)^{2}}{v^{3}} \rho_{\infty} \approx 2 \times 10^{-17} M_{\odot} / \mathrm{yr}$

and an easily possible variation of a factor of 50 up and down due to variations of relative velocity $v$ or hydrogen density $\rho_{\infty}$ could explain all observations.

While we believe that this paradigm shift from "two-phase" to continuous accretion combined with heavy element diffusion is supported by our new observations and the simple arguments above, many questions remain open. We will probably in the near future understand much better the detailed structure of the LISM through work along the lines of Redfield \& Linsky $(2002,2004)$ and Lehner et al. (2003). The calculation of diffusion time scales needs to be extended to hotter DA, to allow us to determine the accretion rates. And finally and 
probably the hardest, the accretion rates under conditions relevant for white dwarfs need more study. While it is not possible to see this in accretion on white dwarfs, it is well established that at least in some and probably all helium-rich white dwarfs the accretion of hydrogen is suppressed by large factors compared to accretion of metals. This points to a preferential accretion of dust grains, a complication which likely needs to be taken into account for a correct derivation of accretion rates.

Acknowledgements. We thank T. Lisker and C. Karl for performing the reduction of the UVES survey spectra. D.K. and B.V. acknowledge support for this work through grant Ko738/21-1 from the Deutsche Forschungsgemeinschaft. R.N. acknowledges support by a PPARC Advanced Fellowship.

\section{References}

Aannestad, P. A., Kenyon, S. J., Hammond, G. L., \& Sion, E. M. 1993, AJ, 105, 1033

Alcock, C., \& Illarionov, A. 1980, ApJ, 235, 541

Dupuis, J., Fontaine, G., Pelletier, C., \& Wesemael, F. 1992, ApJS, 82, 505

Dupuis, J., Fontaine, G., Pelletier, C., \& Wesemael, F. 1993a, ApJS, 84,73
Dupuis, J., Fontaine, G., \& Wesemael, F. 1993b, ApJS, 87, 345

Friedrich, S., Koester, D., Heber, U., Jeffery, C. S., \& Reimers, D. 1999, A\&A, 350, 865

Friedrich, S., Koester, D., Christlieb, N., Reimers, D., \& Wisotzki, L. 2000, A\&A, 363, 1040

Holberg, J. B., Barstow, M. A., \& Green, E. M. 1997, ApJ, 474, L127

Koester, D. 1976, A\&A, 52, 415

Koester, D., Provencal, J., \& Shipmann, H. L. 1997, A\&A, 320, L57

Koester, D., Napiwotzki, R., Christlieb, N., et al. 2001, A\&A, 378, 556

Lehner, N., Jenkins, E. B., Gry, C., et al. 2003, ApJ, 595, 858

McCook, G. P., \& Sion, E. M. 1999, ApJS, 121, 1

Napiwotzki, R., Christlieb, N., Drechsel, H., et al. 2001, Astron. Nachr., 322, 411

Napiwotzki, R., Christlieb, N., Drechsel, H., et al. 2003, The Messenger, 112, 25

Redfield, S., \& Linsky, J. L. 2002, ApJS, 139, 439

Redfield, S., \& Linsky, J. L. 2004, ApJ, 602, 776

Wesemael, F. 1979, A\&A, 77, 354

Wolff, B., Koester, D., \& Liebert, J. 2002, A\&A, 385, 995

Zuckerman, B., Koester, D., Reid, I. N., \& Hünsch, M. 2003, ApJ, 596, 477

Zuckerman, B., \& Reid, I. N. 1998, ApJ, 505, L143 